\title{
(2) OPEN ACCESS \\ Randomised trial of a serious illness decision aid (Plan Well Guide) for patients and their substitute decision- makers to improve engagement in advance care planning
}

\author{
Michelle Howard (D) , 'Dawn Elston, ${ }^{1}$ Sayem Borhan, ${ }^{2}$ Abe Hafid, \\ Neha Arora, ${ }^{1}$ Ruth Forbes, ${ }^{3}$ Carrie Bernard, ${ }^{1,4}$ Daren K Heyland (D) ${ }^{5}$
}

- Additional supplemental material is published online only. To view, please visit the journal online (http://dx.doi. org/10.1136/bmispcare-2021 003040).

For numbered affiliations see end of article.

\section{Correspondence to}

Michelle Howard, Department of Family Medicine, McMaster University Faculty of Health Sciences, Hamilton, Canada; mhoward@mcmaster.ca

Received 15 March 2021 Accepted 10 June 2021

\section{Check for updates}

(c) Author(s) (or their employer(s)) 2021. Re-use permitted under CC BY-NC. No commercial re-use. See rights and permissions. Published by BMJ.

\footnotetext{
To cite: Howard M, Elston D, Borhan S, et al. BMJ Supportive \& Palliative Care Epub ahead of print: [please include Day Month Year]. doi:10.1136/ bmjspcare-2021-003040
}

\section{ABSTRACT}

Objective To evaluate the feasibility and efficacy of a serious illness decision aid (Plan Well Guide) in increasing the engagement of substitute decisionmakers (SDM) in advance care planning (ACP).

Methods This trial was conducted (2017-2019) in outpatient settings in Ontario, Canada, aiming to recruit 90 dyads of patients aged 65 years and older at high risk of needing future medical decisions and their SDM. Participants were randomised to receive the intervention immediately or to a 3-month wait period. The Plan Well Guide was administered to the patient and SDM by a facilitator. Outcomes were change on the validated 17-item SDM ACP Engagement Survey (primary) and 15-item patient ACP Engagement Survey (secondary).

Results Of 136 dyads approached, 58 consented and were randomised and 45 completed the study (28 immediate intervention, 17 delayed intervention). The trial was stopped early because of difficulties with enrolling and following up participants. The mean changes on the SDM ACP Engagement Survey and the patient ACP Engagement Survey favoured the first group but were not statistically significant (mean difference $(\mathrm{MD})=+0.2(95 \% \mathrm{Cl}:-0.3$ to 0.6$)$ and $\mathrm{MD}=+0.4$ (95\% Cl: -0.1 to 0.8$)$, respectively). In a post-hoc subgroup analysis, significant treatment effects were seen in SDMs with a lower-than-median baseline score compared with those at or above the median. Conclusions In this statistically underpowered randomised trial, differences in SDM ACP engagement between groups were small. Further information is needed to overcome recruitment challenges and to identify people most likely to benefit from the Plan Well Guide.

Trial registration number NCT03239639

\section{Key messages}

What was already known?

- Substitute decision-makers have difficulty making medical decisions consistent with their older loved one's wishes.

What are the new findings?

- The Plan Well Guide did not increase advance care planning engagement of substitute decision-makers, except in those least engaged.

What is their significance?

- (a) Clinical: Plan Well Guide may benefit some people.

- (b) Research: Methods are needed to overcome recruitment challenges in older patients.

\section{BACKGROUND RATIONALE}

Many seriously ill older adults, at some point, will lack the capacity to make their own medical treatment decisions. ${ }^{1}$ In the event that a patient becomes incapable of making medical decisions, substitute decision makers (SDM) will be approached to make decisions on the patient's behalf. SDMs report challenges making medical decisions on behalf of loved ones due to lack of a clear understanding of their loved one's values and wishes, which can lead to distress. ${ }^{2}$ In order for the SDM to engage in medical decision-making with the healthcare team in a way that upholds the patient's values and previous wishes, the SDM would ideally have a greater understanding of the patient's values and wishes.

Advance care planning (ACP) is a process that supports adults at any age 
or stage of health in understanding and sharing their personal values, life goals and preferences regarding future medical care. ${ }^{3}$ Studies have shown that previous engagement in ACP by the patient is associated with increased likelihood that a patient's previously expressed wishes will guide medical treatment decisions, ${ }^{4-7}$ increased likelihood of receiving hospice services and reduced likelihood of hospitalisation and provision of intensive treatments (according to patients' wishes) at the end of life. ${ }^{89}$

While ACP has clear benefits to patients and healthcare systems, studies continue to document problems with the quality or quantity of communication and decision-making during serious illness, ${ }^{10}{ }^{11}$ suggesting that ACP has not been undertaken successfully. Patients can express preferences for the use or nonuse of life-sustaining treatments, however they also report considerable decisional conflict about the preference. Furthermore, patients often hold conflicting values from a medical perspective, such as living as long as possible and also minimising invasive treatments. ${ }^{12}$ There is misunderstanding among the public of the benefits and harms of treatments during serious illness such as cardiopulmonary resuscitation ${ }^{13}$ and palliative care ${ }^{14}$ and a lack of understanding of the SDM role. ${ }^{15}$ These common misunderstandings that undermine successful ACP likely contribute to the decisional conflict and regret experienced by some SDM. ${ }^{16}$

The novel decision aid, (the Plan Well Guide tool; www.planwellguide.com), was created to help patients clarify their authentic values and be truly informed about the medical treatment options in the context of serious illness. In a randomised trial of the Plan Well Guide in primary care, some aspects of decisional quality improved while reducing the physician's time to accomplish goals of care decisions. ${ }^{17}$ Although one would imagine that improved decision-making would have a positive effect on the patient's SDM, this trial did not investigate the effect on SDMs.

We conducted a randomised trial to evaluate the efficacy of the Plan Well Guide used with a patient in the presence of the patient's SDM, in increasing the SDM's ACP engagement behaviours. The secondary objectives were to evaluate the efficacy of the Plan Well Guide to increase ACP engagement behaviours of the patient. We were also interested in feasibility of recruiting SDMs in the community for ACP research.

\section{METHODS}

This was a parallel group randomised controlled trial where participants were randomised to immediate intervention or delayed intervention (control group) in a 1:1 allocation ratio. Patients allocated to the delayed intervention received the intervention after they had completed the 3-month outcome assessment. The protocol has been previously published. ${ }^{18}$

\section{Recruitment}

Participants were enrolled from September 2017 to October 2019 from nine outpatient settings in Ontario, Canada, including family practice, cardiology, cancer pain and symptom management and chronic obstructive pulmonary disease clinics (table 1). For most participants, physicians identified the patient as potentially eligible at a visit and referred them to the research assistant on site. In two family practices, to boost recruitment the physician also reviewed the electronic medical record and contacted potentially eligible patients by telephone. SDMs were invited at the time of the clinic visit with the patient or they were contacted by the research assistant after receiving permission from the patient.

\section{Participants and enrolment criteria}

Eligible participants were dyads of adult patients aged 65 years and older who could identify an SDM willing to participate and the patient's identified SDM aged 18 years or older. Clinics were asked to identify adult patients considered 'high-risk' for probability of a decline in health that may require hospitalisation, for example, based on age, comorbidities, change in health status or recent hospitalisations. ${ }^{19} 20$

Additional eligibility criteria included being cognitively able to consent to research (as perceived by the referring clinician's judgement, and confirmed by the research staff obtaining consent), able to communicate in English and having adequate hearing and vision (with aids) to be able to interact with the research staff. Exclusion criteria included inability to communicate in English.

\section{Data collection}

The baseline outcome measurement was administered after randomisation in both groups to accommodate the request of many participants to be interviewed in person rather than by telephone, and to receive the intervention at the same time (for those randomised to

Table 1 Numbers of dyads (patient and substitute decision-maker) approached, randomised and completed, by study site

\begin{tabular}{lccc}
\hline Site & No of dyads approached & No of dyads randomised & No of dyads completed follow-up \\
\hline Family practices & 136 & 36 & 26 \\
Cancer outpatient clinic & 23 & 10 & 5 \\
Heart failure clinic & 64 & 17 & 12 \\
Chronic obstructive pulmonary disease clinic & 7 & 3 & 2 \\
\hline
\end{tabular}


the immediate intervention). Therefore, participants were aware of their group allocation at the time of the baseline measurements.

The follow-up measure was administered either by telephone in the immediate intervention group or at the in-person study visit at the delayed intervention appointment.

\section{Randomisation, allocation, concealment and blinding}

Randomisation was blocked using variable block size, and concealed using sequentially numbered opaque envelopes. Participants could not be blinded. Outcome measurement was not blinded. Investigators were blinded to group allocation of the participants and the data analyst was blinded.

\section{Study intervention}

Details of the Plan Well Guide slide show version used in this study (slides with voiceover) have been described previously. ${ }^{17} \mathrm{~A}$ trained research nurse who had practiced the intervention in mock sessions, ran the interactive Plan Well Guide for the patient and SDM on a laptop computer and completed the structured paper-based values clarification and preference questions with the patient when prompted by the decision aid. The research nurse could assist the patient through their deliberations about the values and preferences questions and review the information from sections of the tool as needed until the patient was comfortable expressing a preference, but did not provide advice about personal medical decisions.

The Plan Well Guide (1) describes the difference between serious illness and terminal care, and the significance of making decisions under conditions of uncertainty, (2) defines personal values, why these are important in decision-making and how tradeoffs are sometimes required, (3) provides education about different approaches to care in hospital and the outcomes of various treatment options (resuscitative/ intensive care, medical care, comfort care) in probabilistic terms with visual aids and (4) guides the patient through a values and preference elicitation process, explicitly linking values to preferences using rating scales and a grid (see www.planwellguide.com). The grids are used to provide structured guidance to the patient helping them link their stated values to reasonable treatment preferences. If the patient indicates a preference for cardiopulmonary resuscitation (CPR), a $\mathrm{CPR}$ information video is shown describing the benefits and risks of this procedure. ${ }^{21}$ If the patient feels there is discordance in the treatment preference highlighted on the grids (eg, their values regarding prolonging life and willingness to tolerate interventions are inconsistent with treatment preference aligned on the grid), the research nurse reviews the patient's values, and which values are most important to determine if they would like any changes. The final step is to elicit a treatment preference for the use of life-sustaining treatments. A previously published taxonomy describing different levels of the use or non-use of life-sustaining treatments is used. ${ }^{22} 23$

\section{Usual care}

Participants randomised to delayed intervention were contacted by phone for in-person second baseline measures at 12 weeks and were then given the intervention.

At baseline, to ensure all participants received current publicly available information about ACP, patients in both groups received a booklet on ACP created by the provincial Hospice Palliative Care Association, which describes the provincial legal context of ACP but does not duplicate the content of the Plan Well Guide.

\section{Outcome measures}

The primary outcome was the overall score on a validated 17-item 'SDM ACP Engagement Survey'. The survey is based on a previously validated patient ACP Engagement Survey ${ }^{24}$ and was adapted for SDMs. ${ }^{25}$ The patient ACP Engagement Survey is based on stages of behaviour change, meaning that questions ask about processes that precede actual actions (including knowledge, contemplation, self-efficacy, readiness) as well as actions, such as discussing one's wishes with the SDM and documenting wishes. Within each domain, questions pertain to Behaviour Change Processes on 5 -point Likert scales where a score of 1 indicates the lowest level of engagement and 5 indicates the highest. Actions are based on questions of whether an ACP action such as asking someone to be an SDM or documenting wishes, was completed. ${ }^{24}$ Several versions of varying lengths have been validated. ${ }^{26}$ The 17 -item SDM ACP Engagement Survey was created to measure the impacts of ACP tools and interventions on SDMs and elicits knowledge, contemplation, self-efficacy, readiness and actions with respect to the SDM role. In the initial validation study, internal consistency reliability was $>0.9$ and the proportion of variance explained was $91 \%{ }^{25}$ There is an overall score and subscale scores for the aforementioned domains. At the time of this trial, the SDM ACP Engagement Survey had not been studied for its responsiveness to an intervention.

Secondary SDM outcomes were changes in the subscale scores of the SDM ACP Engagement Survey. Change in ACP engagement of the patient was also a secondary outcome, measured by the 15 -item version of the aforementioned patient ACP Engagement Survey (internal consistency reliability 0.92, Pearson correlation with original full-length survey 0.91$){ }^{26}$

Information collected to describe participants included age, sex, education level, health literacy and a modified Clinical Frailty Scale (CFS) which excluded the highest category of terminal illness. ${ }^{27}$ The CFS was shown to each dyad, input was sought from the patient and consensus agreed on. Additionally, for SDMs, 
their relationship to the patient and whether they had been formally asked by the patient to be the SDM were asked.

\section{Follow-up}

The outcome assessment took place 12 weeks after the intervention (or baseline interview in the delayed intervention group). This timing was chosen allow time for the patient and SDM to reflect and further discussvalues and preferences, to enhance the SDM's perception of confidence and readiness in the SDM role.

On average, follow-ups were completed in 14 weeks (minimum: 6 weeks; maximum: 21 weeks). Between 3 and 10 attempts were made, as needed, to reach the patient and SDM for follow-up. If only one member of the dyad completed follow-up, their data was used as appropriate for analyses of primary or secondary outcomes.

\section{Sample size and statistical analysis}

The sample size was based on the mean difference between groups on the change in score for the SDM ACP Engagement Survey. The target sample size to detect an effect size (ratio of difference between groups to standard deviation [SD]) of at least 0.6 (small, $0.20-0.49$; moderate, $0.5-0.79$; large, $\geq 0.80^{28}$ ) for the primary outcome with $80 \%$ power at a twosided alpha $=0.05$ is $16 /\left(0.6^{2}\right)$ was 45 per group. A moderate effect size was expected based on previous studies testing the effect of a computer-based ACP tool in similar populations. ${ }^{29} 30$

The primary analysis was performed using an intention-to-treat approach. For the intention-totreat analysis, multiple imputation was used, assuming missing data followed a missing at random pattern. In total, five data sets were imputed. The pooled effect estimates in terms of MD along with 95\% confidence intervals (CI) were reported. Sensitivity analysis was done using the per-protocol approach.

The overall scores of the SDM ACP Engagement Survey and the patient ACP Engagement Survey were calculated as the mean of all questions on the 5-point response scales for the entire survey. ACP actions were elicited within the 'readiness' questions, which includes responses of 'I have never thought about it'; 'I have thought about it but I am not ready to do it'; 'I am planning to do it in the next 6 months'; 'I am definitely planning to do it in the next 30 days'; and, 'I have already done it'. If a patient reported 'I have already done it', they were considered to have completed that action for that behaviour.

The primary and secondary outcomes were analysed as the difference between groups in the change from baseline to the 3-month follow-up measurement (after the intervention in the immediate group and a second baseline measurement immediately before receiving intervention in the delayed group). We used multiple linear regression adjusted for the site of recruitment to analyse the continuous outcomes. We conducted a post-hoc subgroup analysis of the outcomes according to whether the baseline SDM ACP Engagement Survey score was above versus equal to or below the median of all study participants.

All statistical tests were two-sided at the level of significance 0.05 . All analyses were conducted using the software R V.4.0.3. ${ }^{31}$

\section{RESULTS}

Two-hundred forty patients and 136 SDM were identified and consented to be contacted by a researcher or were approached directly at a clinic visit (figure 1). Of these, 104 patients and 49 SDM declined to participate, and the remainder could not be reached to discuss the study (eg, no answer, calls not returned). Sixty-six dyads of a patient and their SDM provided consent and were enrolled, however eight withdrew before randomisation and baseline measurement.

Anecdotally, the main reasons for not participating were lack of interest on the part of the patient or SDM due to the perception that ACP had already been undertaken through completion of wills, funeral plans and so on. In-person recruitment was done only on days when a research assistant could be present and when convenient for the clinic. New clinics were added over time to boost recruitment. When the research assistant began noticing the same patients returning for visits over time, they felt that recruitment had been 'saturated' at that site.

Thirty-four dyads were randomised to the immediate intervention group and 24 to the delayed intervention group. Complete follow-up measurement was obtained for $28(82.4 \%)$ dyads in the immediate intervention group and 17 dyads (70.8\%) in the delayed intervention group. Reasons for loss to follow-up mainly related to worsening illness, and in the delayed intervention group lack of interest in completing the second baseline survey and receiving the intervention (figure 1).

The mean age of patient participants at baseline in the immediate and delayed intervention groups was 76 and 74 years, and $50 \%$ and $58 \%$ were women (table 2). The mean age of SDM in both groups was 66 years. In the immediate and delayed intervention groups, $71 \%$ and $63 \%$ were the patient's spouse, respectively. On average, the intervention took $70 \mathrm{~min}$ (minimum $50 \mathrm{~min}$, maximum $140 \mathrm{~min}$ ) to complete.

The mean score on the SDM ACP Engagement Survey at baseline was $3.7(\mathrm{SD}=0.8)$ in the immediate intervention group and $3.6(\mathrm{SD}=0.8)$ in the delayed intervention group. Scores in both groups increased: the mean difference was 0.1 in favour of the immediate intervention group (95\% CI: -0.4 to $0.6 ; \mathrm{p}=0.64$ ) (figure 2). For the subscales of knowledge, contemplation, self-efficacy and readiness, all scores increased to a greater extent in the immediate group than the 


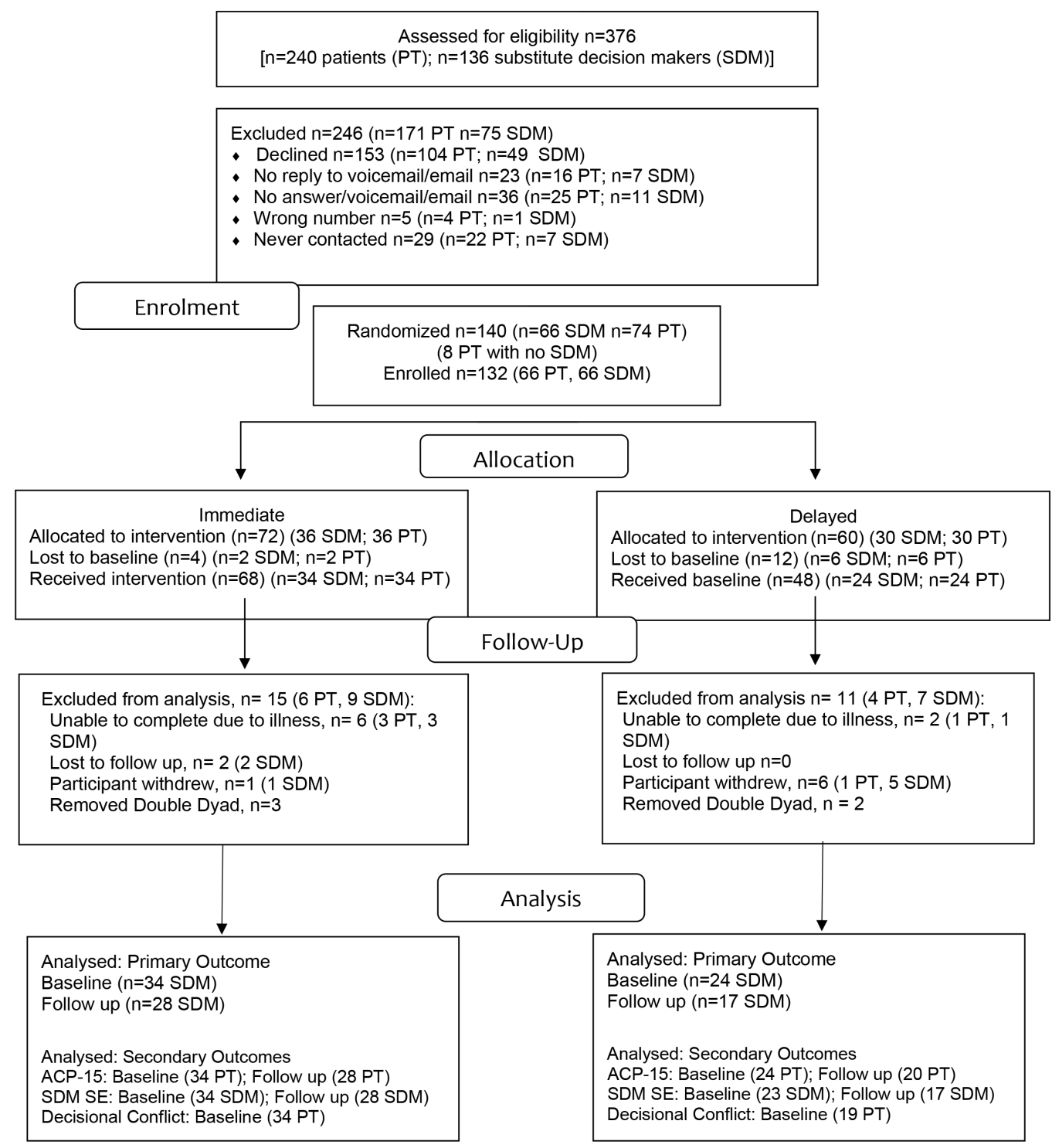

Figure 1 Consolidated Standards of Reporting Trials flow diagram. ACP, advance care planning.

delayed group, however none of the mean differences were statistically significant. The greatest difference in the increase between groups was for the readiness subscale (mean difference $=0.3 ; 95 \% \mathrm{CI}-0.3$ to 1.0 , $\mathrm{p}=0.32$ ). The results were similar after adjusting for study site and in the per-protocol analyses unadjusted and adjusted for study site (online supplemental file 1).

The overall mean score on the patient ACP Engagement Survey at baseline was $3.9(\mathrm{SD}=0.7)$ in the immediate intervention group and $3.7(\mathrm{SD}=0.7)$ in the delayed intervention group. The mean scales in both groups increased: the mean difference was 0.4 in favour of the immediate intervention group $(95 \% \mathrm{CI}$ : -0.1 to $0.8 ; p=0.11$ ) (figure 3 ). The subscale with the largest difference in change between groups was readiness (mean difference $=0.6,95 \% \mathrm{CI}$ : -0.15 to 1.3 ; $\mathrm{p}=0.12)$.

In the post-hoc subgroup analysis, there were 18 immediate intervention and 12 delayed intervention SDMs at or below the median at baseline (figure 4).
In this subgroup, the overall SDM ACP Engagement Survey score increase was statistically significantly greater for the immediate than the delayed intervention group overall (mean difference $=0.6,95 \% \mathrm{CI}$ : 0.03 to $1.2, p=0.04)$, and for the contemplation subscale (mean difference $=0.4,95 \%$ CI: 0.05 to 0.8 , $\mathrm{p}=0.03)$.

\section{DISCUSSION}

In this randomised trial in 45 SDMs of older adult patients, enrolment was stopped early because of recruitment difficulties. Within the small data set accrued, the use of the Plan Well Guide serious illness decision aid did not result in statistically significant differences in SDM engagement in ACP behaviours compared with a control group who received usual care. Patient engagement in ACP behaviour change processes and actions also did not change more in the intervention group compared with the control group. Scores on the measures increased in both groups and 
Table 2 Baseline demographic characteristics of patients and substitute decision-makers in the trial

Delayed

Immediate intervention intervention group $(n=34)$ group $(n=24)$

\section{Patients}

\begin{tabular}{|c|c|c|}
\hline Age, mean (SD) & $76(8)$ & $74(7)$ \\
\hline Female, n (\%) & $17(50)$ & $14(58)$ \\
\hline $\begin{array}{l}\text { Married or living as } \\
\text { married, } \mathrm{n}(\%)\end{array}$ & $27(79)$ & $20(83)$ \\
\hline University degree, $\mathrm{n}(\%)$ & $16(47)$ & $9(38)$ \\
\hline $\begin{array}{l}\text { Self-rated health fair or } \\
\text { poor, } n(\%)\end{array}$ & $6(18)$ & $6(25)$ \\
\hline $\begin{array}{l}\text { Health literacy, } n(\%) \\
\text { Need help sometimes, } \\
\text { often, always }\end{array}$ & $2(6)$ & $2(8)$ \\
\hline $\begin{array}{l}\text { Frailty score, } \mathrm{n}(\%) \\
\text { Vulnerable or higher } \\
\text { (category 4) }\end{array}$ & $6(18)$ & $7(29)$ \\
\hline \multicolumn{3}{|l|}{ Substitute decision-makers } \\
\hline Age (mean, SD) & $66(12)$ & $66(10)$ \\
\hline Female, n (\%) & $23(72)$ & $14(58)$ \\
\hline \multicolumn{3}{|l|}{$\begin{array}{l}\text { Relationship to patient, } \\
\mathrm{n}(\%)\end{array}$} \\
\hline Spouse & $24(71)$ & $15(63)$ \\
\hline Child & $9(27)$ & $7(29)$ \\
\hline Other relative or friend & $1(3)$ & $2(8)$ \\
\hline University degree, n (\%) & $18(54)$ & $13(54)$ \\
\hline $\begin{array}{l}\text { Have been a medical } \\
\text { decision-maker before, } \\
\mathrm{n}(\%)\end{array}$ & $8(24)$ & $9(38)$ \\
\hline $\begin{array}{l}\text { Have been formally asked } \\
\text { by patient to be medical } \\
\text { decision-maker, } \mathrm{n}(\%)\end{array}$ & $21(62)$ & $15(63)$ \\
\hline
\end{tabular}

our expected effect size was not achieved. In a post-hoc analysis, there was a statistically significant difference between groups for overall SDM ACP engagement and for the subscale of contemplation, among the subgroup of SDM who had a baseline below the median overall score.

The Plan Well Guide encourages patients to share the values and preference elicitation exercise with their

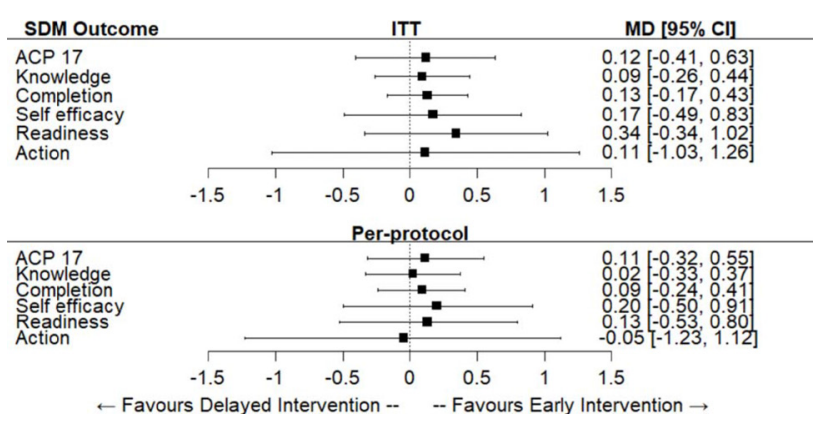

Figure 2 Mean between-group differences and 95\% Cls for the change in the substitute decision-maker (SDM) advance care planning (ACP) Engagement Survey overall score (ACP 17) and subscale scores. MD, mean difference; ITT, intention-totreat.

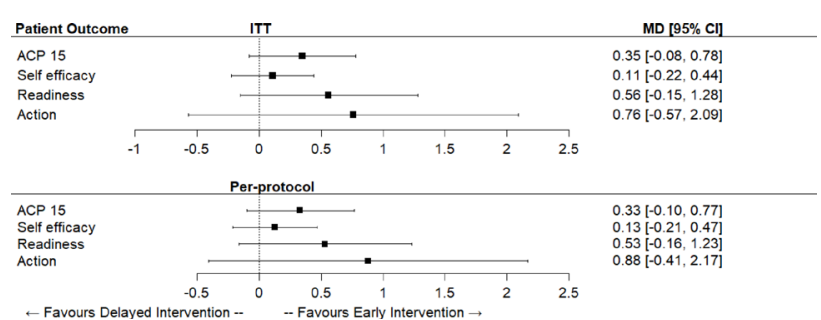

Figure 3 Mean between-group differences and 95\% Cls for the change in patient advance care planning (ACP) Engagement Survey overall score (ACP 15) and subscale scores. MD, mean difference; ITT, intention-to-treat.

SDM. We hypothesised that the presence of the SDM while the patient works through the tool would lead to changes in behaviour change processes of the SDM including knowledge, contemplation, self-efficacy, readiness and actions. There may be several explanations for the lack of detectable differences, besides the underpowered statistical tests due to small sample size. Given the nature of the patient population, participants may have already had conversations about serious illness decision-making. On a contemplation question such as how often the SDM had thought about talking with their loved one about wishes, a response of three is 'a few times' and four is 'several times'. Given that most mean subscale scores on the survey were above 3.5 of a possible 5 at baseline, there may have been little room for improvement. Another explanation may be that SDMs were often present with the patient when responding to the survey and they may have been reluctant to admit lack of readiness for their role.

Our study used the SDM ACP Engagement Survey as an outcome measure, and while some of the psychometric properties have been assessed, it has not been evaluated for responsiveness to the Plan Well Guide intervention. Previous studies of interventions to prepare SDM for decision-making involvement have used SDM decision-making confidence and dyad congruence on preference for use of life-sustaining treatments. ${ }^{32}$ In an international consensus panel of important outcomes

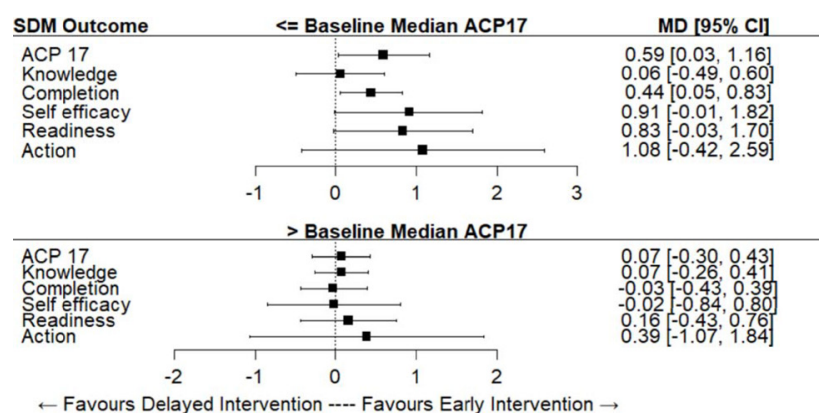

Figure 4 Mean between-group differences and 95\% Cls for the change in the substitute decision-maker (SDM) advance care planning (ACP) Engagement Survey overall score (ACP 17) and subscale scores: subgroup analysis by at or below baseline median versus above baseline median (adjusted for study site, no imputation for missing data). MD, mean difference. 
of ACP, deciding on and sharing values and care preferences with the SDM were voted to be among the most important outcomes. ${ }^{33}$ These outcomes are upstream of actual decision-making quality such as dyad congruence. Therefore, it is important to evaluate whether ACP interventions improve SDM processes of preparation for decision-making.

Recruiting and retaining patients with significant health concerns and their SDM in the community through outpatient clinics was challenging. Poor health and mobility problems have been reported to be barriers to research participation in frail elderly people. ${ }^{34}$ We were not able to recruit the SDM of all patients who were potentially interested. Other studies have reported similar challenges recruiting and retaining patient and SDM dyads in studies of end-of-life communication. ${ }^{35-37}$ Shields et al reported that one-third of consenting patients could not be enrolled in a trial of dyads because the surrogate did not consent. ${ }^{37}$ More understanding is needed on the best way to recruit older patient and SDM dyads for ACP studies because of the importance of the SDM's role. There were more losses to follow-up in the delayed intervention group due to worsening health and lack of interest. A shorter follow-up period should be considered for future studies in this population.

\section{Limitations}

This study has limitations. Most notably, recruitment was lower than expected and the study was statistically underpowered. The recruitment challenges related mainly to SDMs, who were more difficult to reach and engage compared with patients. Some deviations were required from the published protocol, such as conducting the baseline measure after randomisation, to ensure participant recruitment and retention. Nevertheless, baseline measures between groups were similar, and the small magnitude of difference in outcomes suggests that there was no bias towards overestimating effects. The Plan Well Guide has not been translated to languages other than English or adapted for varying cultures, therefore study participants may represent the typical Caucasian English-speaking population as found in many previous studies of ACP. ${ }^{38}$ Models of adapting ACP tools for other populations exist $^{39}$ and should be considered in the future.

\section{CONCLUSIONS}

In this statistically underpowered trial that was stopped early, differences between groups were small, however there was a larger and statistically significant effect in SDM participants with lower baseline ACP engagement. Further information is needed to overcome recruitment challenges and to identify people most likely to benefit from the Plan Well Guide.

\section{Author affiliations}

${ }^{1}$ Department of Family Medicine, McMaster University Faculty of Health Sciences, Hamilton, Ontario, Canada
${ }^{2}$ Health Research Methods, Evidence, and Impact, McMaster University Faculty of Health Sciences, Hamilton, Ontario, Canada

${ }^{3}$ School of Nursing, McMaster University Faculty of Health Sciences, Hamilton, Ontario, Canada

${ }^{4}$ Department of Family and Community Medicine, University of Toronto Faculty of Medicine, Toronto, Ontario, Canada

${ }^{5}$ Department of Critical Care Medicine, Queen's University Faculty of Health Sciences, Kingston, Ontario, Canada

Twitter Michelle Howard @mhoward101

Contributors $\mathrm{MH}$ and $\mathrm{DKH}$ conceived of the study. $\mathrm{MH}$ wrote the paper. RF, DE, AH and NA collected data. $\mathrm{MH}, \mathrm{CB}, \mathrm{SB}$, $\mathrm{DE}, \mathrm{AH}, \mathrm{RF}$ and NA contributed to interpretation of results. SB conducted statistical analyses. $\mathrm{MH}, \mathrm{CB}, \mathrm{SB}, \mathrm{DE}, \mathrm{RF}, \mathrm{AH}$, $\mathrm{NA}$ and DKH critically revised the manuscript and approved the final version for submission.

Funding This work was supported by the Canadian Institutes of Health Research grant number PHE-135930. The funder had no role in the design of the study, collection, management, analysis or interpretation of data, writing of any reports or the decision to submit any report for publication.

Competing interests None declared.

Patient consent for publication Not required.

Ethics approval Ethics approval was obtained by the Hamilton Integrated Research Ethics Board (number 2017-3714-GRA) on 26 September 2017. All participants provided written informed consent.

Provenance and peer review Not commissioned; externally peer reviewed.

Data availability statement Data from this study are available upon reasonable request.

Open access This is an open access article distributed in accordance with the Creative Commons Attribution Non Commercial (CC BY-NC 4.0) license, which permits others to distribute, remix, adapt, build upon this work noncommercially, and license their derivative works on different terms, provided the original work is properly cited, appropriate credit is given, any changes made indicated, and the use is noncommercial. See: http://creativecommons.org/licenses/by-nc/4. $0 /$.

\section{ORCID iDs}

Michelle Howard http://orcid.org/0000-0001-8127-5492

Daren K Heyland http://orcid.org/0000-0002-6440-4616

\section{REFERENCES}

1 Raymont V, Bingley W, Buchanan A, et al. Prevalence of mental incapacity in medical inpatients and associated risk factors: cross-sectional study. Lancet 2004;364:1421-7.

2 Fried TR, Zenoni M, Iannone L, et al. Engagement in advance care planning and surrogates' knowledge of patients' treatment goals. J Am Geriatr Soc 2017;65:1712-8.

3 Sudore RL, Lum HD, You JJ, et al. Defining advance care planning for adults: a consensus definition from a multidisciplinary Delphi panel. J Pain Symptom Manage 2017;53:821-32.

4 Hammes BJ, Rooney BL. Death and end-of-life planning in one midwestern community. Arch Intern Med 1998;158:383-90.

5 Silveira MJ, Kim SYH, Langa KM. Advance directives and outcomes of surrogate decision making before death. N Engl J Med Overseas Ed 2010;362:1211-8.

6 Detering KM, Hancock AD, Reade MC, et al. The impact of advance care planning on end of life care in elderly patients: randomised controlled trial. BMJ 2010;340:c1345.

7 Metzger M, Song M-K, Ward S, et al. A randomized controlled pilot trial to improve advance care planning for LVAD patients and their surrogates. Heart Lung 2016;45:186-92. 
8 Teno JM, Fisher ES, Hamel MB, et al. Medical care inconsistent with patients' treatment goals: association with 1-year Medicare resource use and survival. J Am Geriatr Soc 2002;50:496-500.

9 Houben CHM, Spruit MA, Groenen MTJ, et al. Efficacy of advance care planning: a systematic review and meta-analysis. $J$ Am Med Dir Assoc 2014;15:477-89.

10 You JJ, Dodek P, Lamontagne F, et al. What really matters in end-of-life discussions? Perspectives of patients in hospital with serious illness and their families. CMAJ 2014;186:E679-87.

11 Khandelwal N, Curtis JR, Freedman VA, et al. How often is end-of-life care in the United States inconsistent with patients' goals of care? J Palliat Med 2017;20:1400-4.

12 Heyland DK, Heyland R, Dodek P, et al. Discordance between patients' stated values and treatment preferences for end-of-life care: results of a multicentre survey. BMJ Support Palliat Care 2017;7:292-9.

13 Jones GK, Brewer KL, Garrison HG. Public expectations of survival following cardiopulmonary resuscitation. Acad Emerg Med 2000;7:48-53.

14 Roulston E. Canadians' views on palliative care. J Palliat Med 2018;21:S-9-S-14.

15 Wong MKY, Jiang M, Medor MC, et al. Does the public feel prepared to be substitute decision-makers? gaps in preparedness and support for a high school curriculum: a national survey. Age Ageing 2021;50:242-7.

16 Hickman RL, Daly BJ, Lee E. Decisional conflict and regret: consequences of surrogate decision making for the chronically critically ill. Appl Nurs Res 2012;25:271-5.

17 Heyland DK, Heyland R, Bailey A, et al. A novel decision aid to help plan for serious illness: a multisite randomized trial. CMAJ Open 2020;8:E289-96.

18 Howard M, Slaven M, Bernard C, et al. Decision support intervention (plan well guide) for patients and their substitute decision-makers to improve engagement in advance care planning: protocol for a randomised trial. BMJ Open 2019;9:e027897.

19 Downar J, Goldman R, Pinto R, et al. The "surprise question" for predicting death in seriously ill patients: a systematic review and meta-analysis. CMAJ 2017;189:E484-93.

20 Bernacki RE, Block SD, American College of Physicians High Value Care Task Force. Communication about serious illness care goals: a review and synthesis of best practices. JAMA Intern Med 2014;174:1994-2003.

21 You JJ, Swinton M, Mantle C, et al. P043 effect of a video decision aid on clinical decision-making about cardiopulmonary resuscitation. J Pain Symptom Manage 2016;52:e77.

22 Heyland DK, Dodek P, Lamontagne F, et al. The development and validation of a questionnaire to audit advance care planning (ACP). BMJ Support Palliat Care 2012;2:175.3-6.

23 Heyland DK, Ilan R, Jiang X, et al. The prevalence of medical error related to end-of-life communication in Canadian hospitals: results of a multicentre observational study. BMJ Qual Saf 2016;25:671-9.
24 Sudore RL, Stewart AL, Knight SJ, et al. Development and validation of a questionnaire to detect behavior change in multiple advance care planning behaviors. PLoS One 2013;8:e72465.

25 Van Scoy LJ, Day AG, Howard M, et al. Adaptation and preliminary validation of the advance care planning engagement survey for surrogate decision makers. J Pain Symptom Manage 2019;57:980-8.

26 Sudore RL, Heyland DK, Barnes DE, et al. Measuring advance care planning: optimizing the advance care planning engagement survey. J Pain Symptom Manage 2017;53:669-81.

27 Rockwood K, Song X, MacKnight C, et al. A global clinical measure of fitness and frailty in elderly people. CMAJ 2005;173:489-95.

28 Cohen J. Statistical power for the behavioral sciences. Hillsdale, NJ: Erlbaum, 1988.

29 Lum HD, Barnes DE, Katen MT, et al. Improving a full range of advance care planning behavior change and action domains: the prepare randomized trial. J Pain Symptom Manage 2018;56:575-81.

30 Howard M, Robinson CA, McKenzie M, et al. Effect of an interactive website to engage patients in advance care planning in outpatient settings. Ann Fam Med 2020;18:110-7.

31 R Foundation for Statistical Computing. R: a language and environment for statistical computing. Vienna, Austria, 2020.

32 McMahan RD, Tellez I, Sudore RL. Deconstructing the complexities of advance care planning outcomes: what do we know and where do we go? A scoping review. J Am Geriatr Soc 2021;69:234-44.

33 Sudore RL, Heyland DK, Lum HD, et al. Outcomes that define successful advance care planning: a Delphi panel consensus. $J$ Pain Symptom Manage 2018;55:245-55.

34 Provencher V, Mortenson WB, Tanguay-Garneau L, et al. Challenges and strategies pertaining to recruitment and retention of frail elderly in research studies: a systematic review. Arch Gerontol Geriatr 2014;59:18-24.

35 Fischer DJ, Burgener SC, Kavanaugh K, et al. Conducting research with end-of-life populations: overcoming recruitment challenges when working with clinical agencies. Appl Nurs Res 2012;25:258-63.

36 Steinhauser KE, Clipp EC, Hays JC, et al. Identifying, recruiting, and retaining seriously-ill patients and their caregivers in longitudinal research. Palliat Med 2006;20:745-54.

37 Shields A-M, Park M, Ward SE, et al. Subject recruitment and retention against quadruple challenges in an intervention trial of end-of-life communication. J Hosp Palliat Nurs 2010;12:312-8

38 Lin C-P, Evans CJ, Koffman J, et al. The conceptual models and mechanisms of action that underpin advance care planning for cancer patients: a systematic review of randomised controlled trials. Palliat Med 2019;33:5-23.

39 Sudore RL, Schillinger D, Katen MT, et al. Engaging diverse English- and Spanish-speaking older adults in advance care planning: the prepare randomized clinical trial. JAMA Intern Med 2018;178:1616-25. 